\title{
A review of hydrogen and natural gas addition in diesel HCCI engines
}

\begin{abstract}
Homogeneous charge compression ignition (HCCI) engine uses a relatively new mode of combustion technology. In principle, there is no spark plug or injector to assist the combustion, and the combustion auto-ignites in multiple spots once the mixture has reached its chemical activation energy. The challenges in developing HCCI engines are the difficulties in: controlling the auto-ignition of the mixture and the heat release rate at high load operations, achieving a cold start, meeting emission standards and controlling knock. At low engine speeds, early auto-ignition can occur, possibly leading to knocking, while late auto-ignition at high engine speeds will make HCCI susceptible to misfire. Hydrogen greatly reduces emissions levels but with reduced power. However, when hydrogen is combined with diesel in dual-fuel mode, low NOx, CO and particulate matter (PM) emissions levels can be achieved, and engine efficiency can be increased by 13-16\%. Numerical methods are commonly used to predict HCCI engines' performance (i.e. emissions levels, brake thermal efficiency and combustion phasing), which is cost-effective compared to solely relying on experimentation. The multi-zone method promises better simulation results compared to the single-zone model by combining detailed chemical kinetics with simplified 3D modeling so that turbulence and inhomogeneity in the mixture are considered; good agreement between simulations and experiments have been achieved. Specific strategies used in the experimental method (e.g. fuel additives, inlet air heating, inlet air pressurizing, exhaust gas recirculation (EGR) and injection methods), and numerical method (e.g. single-zone and multi-zone models, mixing model, turbulence model and multi-dimensional model), and other issues associated with HCCI engines are discussed in this paper.
\end{abstract}

Keyword: HCCI; Engine; Hydrogen; Natural gas; Diesel; Numerical; Chemical kinetic 